Cahiers de recherches médiévales

\title{
Mourir de laide mort despite
}

L'empoisonnement d'Alexandre dans la littérature française médiévale

Hélène Bellon-Méguelle

\section{CpenEdition}

Journals

Édition électronique

URL : https://journals.openedition.org/crm/11511

DOI : 10.4000/crm. 11511

ISSN : 1955-2424

Éditeur

Honoré Champion

Édition imprimée

Date de publication : 15 juin 2009

Pagination : 141-160

ISSN : 1272-9752

Référence électronique

Hélène Bellon-Méguelle, « Mourir de laide mort despite », Cahiers de recherches médiévales [En ligne], 17 | 2009, mis en ligne le 15 juin 2012, consulté le 15 décembre 2022. URL : http:// journals.openedition.org/crm/11511; DOI : https://doi.org/10.4000/crm.11511 


\title{
RM
}

\section{Mourir de laide mort despite. L'empoisonnement d'Alexandre dans la littérature française médiévale}

\begin{abstract}
Alexander's sudden and mysterious death by poisoning is an integral part of his vita in medieval French and Latin literature. The conqueror is an ambivalent hero, as ambivalent as the theme of poison in the works about him. The constant threat produced by his encounters with harmful liquids is suggestive of his excessive desire, a vice that is compared with venom in Alexandrian literature. The conqueror's unquenchable thirst is a metaphor for his immoderate and ever-unsatisfied desire. Alexander is forced to wander in the deserts of Asia Minor and India in search of fresh water. When he does find water, his expectations are systematically disappointed, as the water more often than not turns out to be toxic. But Alexander's misfortune of having to drink bitter water is comparable to the Passion of Christ, and identifies the ancient hero with epic warriors of the "matière de France" such as Roland or Vivien. The hero's poisoning can also be seen as enhancing his status. His foreshadowed death adds a pronounced tragic dimension to his destiny. In his quest for absolute domination, Alexander is fettered by the limitations of his mortal body. Invincible warrior, hero of the unlimited, Alexander is also a terribly human figure who meets in death by poison the limits of his ambition, a metaphysical hero who knows the hour of his death and is reminded of it many times in the course of his journey.
\end{abstract}

Résumé: L'empoisonnement d'Alexandre, mort brutale et sournoise, est un élément indissociable de sa vita dans la littérature française et latine du Moyen Âge. Le conquérant est un héros ambivalent, le motif du poison dans les poèmes qui lui sont consacrés l'est également. La menace constante que constitue pour le personnage la rencontre de liquides délétères est une façon de dénoncer sa trop grande convoitise, vice comparé au venin dans les textes alexandrins. Le désir immodéré et toujours insatisfait du conquérant est métaphorisé par sa soif inextinguible. Alexandre est contraint d'errer dans les déserts d'Asie Mineure ou d'Inde à la recherche d'eau douce. Lorsqu'il approche du but, son attente est systématiquement déçue, l'eau qu'il trouve se révélant le plus souvent toxique. Mais le supplice d'Alexandre contraint de boire de l'eau amère est aussi comparable à la passion du Christ et identifie le héros antique aux guerriers épiques de la matière de France comme Roland ou Vivien. L'empoisonnement du héros peut aussi être perçu comme un élément valorisant de son existence. Cette mort annoncée donne à sa destinée une forte dimension tragique. Dans sa quête de domination absolue, Alexandre est entravée par les limites que lui impose son corps de mortel. Guerrier invincible, héros de l'illimité, Alexandre est aussi un personnage terriblement humain qui trouve dans la mort par poison le terme de son ambition, un héros métaphysique qui connaît l'heure de sa mort et qui en est maintes fois averti tout au long de son parcours.

Mourir empoisonné à Babylone, au faîte de la gloire, dans la ville tant désirée de l'ultime conquête, tel est le sort d'Alexandre dans la littérature française

Cahiers de Recherches Médiévales, 17, 2009 
médiévale. Cette mort violente et prématurée ${ }^{1}$ marque d'un sceau tragique la vita du héros et ce, dès les premiers textes qui lui sont consacrés. Dans la littérature antique toutefois des divergences existent entre tenants de la tradition légendaire essentiellement les auteurs des différentes recensions du Roman d'Alexandre du Pseudo-Callisthène - et ceux de la tradition historique. Pour les premiers, Alexandre est mort empoisonné. Le meurtre, commandité par Antipater, est perpétré par son fils Cassandre qui apporte le poison à Babylone dans le sabot d'une mule et par Iollas, l'échanson du roi, qui, pour se venger d'une correction infligée peu de jours auparavant par Alexandre, n'hésite pas à verser le poison dans la coupe de son maître. Pour la plupart des seconds, les circonstances de la mort du héros ne sont pas claires $^{2}$. Tous s'accordent à dire qu'Alexandre fut emporté par une forte fièvre. Quant à sa cause, maladie ou empoisonnement, ils ne tranchent pas vraiment. $\mathrm{Au} \mathrm{II}$ siècle, Arrien reste sceptique quant à la thèse du crime. Dans l'Anabase, après avoir rappelé la rumeur d'un empoisonnement par Antipater, il se sent obligé d'ajouter : «Je me dois de rapporter ces histoires, afin de ne pas sembler ignorer qu'elles ont été dites, plutôt que parce qu'elles constitueraient un récit digne de foi » (Anabase, VII, 27) ${ }^{3}$. Diodore est plus enclin à soutenir la thèse de l'empoisonnement; il affirme même que les historiographes ont renoncé à parler de ce crime de peur des représailles qu'aurait pu exercer sur eux Antipater, devenu tout puissant en Europe après la mort du Macédonien (XVII, 118.1). Principale autorité pour les auteurs médiévaux, Quinte Curce, s'il ne se prononce pas réellement, donne des arguments qui rendent vraisemblable la thèse du complot ${ }^{4}$ : une dissension existait bien entre Alexandre et Antipater, le roi reprochant à son préfet une ambition excessive; de surcroît, il est dit qu'en Macédoine le Styx aurait charrié un poison mortel (X.1417). Faisant fi des réserves des historiens antiques, les auteurs médiévaux ne remettront plus en cause la thèse de l'empoisonnement. Au Moyen Âge, le motif du poison devient un élément indissociable de la vie d'Alexandre, il hante son parcours et contribue à faire de lui un héros ambivalent. Cette mort, traditionnellement

\footnotetext{
${ }^{1}$ Plusieurs études ont été consacrées à la mort d'Alexandre, notamment : Alexander the Great in the Middle Ages. Ten Studies on the Last Days of Alexander in Literary and Historical Writing, ed. by W. J. Aerts and al., Nijmegen, Alfa Nijmegen (Medievalia Groningana I), 1978 ; G. Cary, The Medieval Alexander, Cambridge, Cambridge University Press, 1956, reprinted 1967, «Alexander's Premature Death, and the Question of Monarchy and Empire », p. 103-105; C. Jouanno, «Le héros et la mort. Épopée, métaphysique et morale dans le Roman d'Alexandre du Pseudo-Callisthène », PRIS-MA, 9/2, Le héros épique I, 1993, p. 197205 ; C. Gaullier-Bougassas, Les Romans d'Alexandre. Aux frontières de l'épique et du romanesque, Paris, Champion, 1998, «La mort d'Alexandre», p. 504-519; F. Collard, Pouvoir et poison. Histoire d'un crime politique de l'Antiquité à nos jours, Paris, Seuil (L'Univers historique), 2007, p. 22-34.

${ }^{2}$ Les historiens actuels s'entendent pour dire qu'Alexandre serait mort d'une crise de paludisme.

${ }^{3}$ Nous citons la traduction de Pierre Savinel parue dans Arrien, Histoire d'Alexandre, Paris, Les éditions de minuit (Arguments), 1984.

${ }^{4}$ Une lacune dans les manuscrits des Histoires nous prive du récit d'un éventuel crime et de la maladie d'Alexandre. Toutefois, Quinte-Curce revient sur les circonstances de la mort du héros au moment du récit des funérailles du roi.
} 
réservée aux puissants ${ }^{5}$, peut être une marque d'infamie ou au contraire valoriser celui qui en est victime ${ }^{6}$. Nous envisagerons ces deux effets paradoxaux en confrontant les différentes versions de la fin du Macédonien dans les œuvres françaises médiévales qui lui sont consacrées. Il nous faudra également réfléchir à l'incidence de cette mort annoncée sur la figure du héros, ce demi-dieu si tragiquement mortel.

\section{Une mort indigne?}

\section{Une erreur politique}

Dans le Roman d'Alexandre d'Alexandre de Paris, celui qui, dans son enfance, ne tolérait d'être nourri que du lait d'une «franche dame» (I, v. 234) meurt par une cruelle ironie en buvant dans la coupe que lui tend la main d'un serf. Lors du banquet qui suit le couronnement d'Alexandre à Babylone, Antipater et Divinuspater dissimulent du venin de serpent sous leurs ongles, tournant sournoisement l'obligation imposée par le roi à ses serviteurs de couper leurs manches jusqu'aux coudes dans la crainte qu'il avait d'être empoisonné :

Li doi serf qui sa mort li orent aportee,

Li uns sist au mengier de la table honoree,

Et li autres servi en la porpre roëe,

Devant le roi demaine tint la cope doree

Qui iert de riches pierres porprise et aornee.

Molt fu la traïson coiement porparlee :

Por ce qu'il ont des bras chascuns la manche ostee,

En l'ongle de ses paus ot l'entosche boutee,

Qant li rois vaut le vin, la coupe a demandee,

Et cil fiert ens ses paus si la li a donee.

Tantost com ot beü, si li art la coree,

Li cuers li muert el ventre s'a la color müee.

(Alexandre de Paris, Le Roman d'Alexandre, IV, v. 147-158)

Dans la branche III, l'empoisonnement est présenté comme l'aboutissement d'un enchaînement de faits relevant du rapport de forces politiques. Alexandre reçoit une lettre d'Olympias qui le met en garde contre Antipater et Divinuspater, vassaux que la mère du roi juge déloyaux et insoumis ; celui-ci décide de les convoquer sur le champ à Babylone pour faire la lumière sur leurs agissements. Les deux hommes,

${ }^{5}$ Cf. F. Collard, Poison et pouvoir, op. cit.

${ }^{6}$ Voir à ce sujet F. Collard, Le crime de poison au Moyen Âge, Paris, PUF (Le Noud gordien), 2003, chapitre VI : «Au-delà du crime : enjeux et usages du poison», part. p. 238-239.

${ }^{7}$ The Medieval French Roman d'Alexandre, volume II : Version of Alexandre de Paris, ed. by E. C. Armstrong, D. L. Buffum, B. Edwards, L. F. H. Lowe, Princeton, Princeton University Press (Elliott Monographs XXXVII), 1937, réed. New York, Kraus Reprints, 1965. 
humiliés et furieux, complotent alors l'assassinat de leur suzerain ${ }^{8}$. Le roi, soucieux de garder la main haute sur toutes les terres qu'il a conquises, n'a pas su s'attirer la bienveillance d'Antipater et de Divinuspater qui se sentent rabaissés et menacés par l'autoritarisme du roi. Ce dernier subit finalement le sort réservé aux mauvais chefs, tel qu'il est présenté par Marcien dans les Voux du paon, interpolation du Roman d'Alexandre écrite au début du $\mathrm{XIV}^{\mathrm{e}}$ siècle $^{9}$ :

Tolir et vilener, et desheritement,

Estre fel et cruël, et parler laidement,

Font haïr leur seigneur et son avancement.

Et quant le sien li héent, je vous demant conment

Il puet ainssi durer ne vivre longuement !

Ou il muert de poison, ou d'enveninmement [...]

(Les Voux du paon, v. 4870-4875)

Dans la branche IV du Roman d'Alexandre, le mobile politique du crime n'est pas rappelé. Les deux hommes semblent n'accomplir leur forfait que parce qu'ils sont des êtres vils et perfides. Tout se passe comme si la seule faute commise par Alexandre était d'avoir fait venir à sa cour deux serfs affranchis ${ }^{10}$ et d'être tombé dans le piège qu'ils lui tendaient. Le roi avait reçu plusieurs mises en garde concernant les hommes de basse extraction. Aristote, dans l'enseignement politique dispensé à son disciple, lui avait bien recommandé : «Que ja sers de put aire n'ait entor lui sovent, / Car maint home en son mort et livré a torment, / Par losenge et par murdre, par enpoisonement. »(I, v. 344-346). Le maître avait réitéré ses conseils dans la branche III : « Se tu ne crois tes sers, ja ne seras honis ; / Ja sers ne sera bons

\footnotetext{
${ }^{8}$ Dans le Voyage d'Alexandre au paradis terrestre, une autre raison est donnée au désir de vengeance des deux vassaux. Alexandre soucieux de récompenser les services de Gracien, lui accorde le fief qu'il désire. Celui-ci demande Tyr et Sidon, les terres d'Antipater et de Divinuspater. Alexandre décide alors de leur reprendre ces possessions en échange de la Chaldée. Pour empêcher cette tractation qu'ils jugent inéquitable et pour éviter l'exil, les deux hommes prennent la décision d'empoisonner le roi. Le narrateur imagine que la ville de Tyr changea de nom en souvenir du forfait : «Por Thir molra li rois, n'en avra garison, / De mort crueuse et sure par amere puison; / Et por celle aventure a or Thir Sur a non : / Surement en fu mors li bons hoirs Phillipon. » (La Prise de Defur and Le Voyage d'Alexandre au paradis terrestre, ed. by L. P. G. Peckham and M. S. La Du, Princeton, Princeton University Press (Elliott Monographs XXXV), 1935, réed. New York, Kraus reprint corporation, 1965, v. 432435).

${ }^{9}$ John Barbour, The Buik of Alexander, ed. by G. Ritchie, 4 vol., Edinburgh-London, William Blackwood and Sons (The Scottish Text Society), 1921-1929.

${ }^{10}$ Rien n'est dit de la condition servile d'Antipater et de Divinuspater dans la branche III du roman où les deux hommes sont présentés comme de nobles barons d'Alexandre. Cette précision est introduite dans la dernière branche et participe du topos du manque de fiabilité des hommes sans naissance. Catherine Gaullier-Bougassas relève cette contradiction dans Les Romans d'Alexandre, op. cit. : «C'est au mépris de l'histoire (Antipater était l'un des Grands de la cour de Philippe) et aussi de la vraisemblance de son propre récit qu'il attribue soudainement cette origine sociale aux deux assassins. » (p. 504).
} 
qui sovent n'est aflis [...] Assés voit on de ciaus qu'ont lor segnors traïs, / Ques ont empoisounés ou as coustiaus murdris ; / Ton conseil ne lor di ne en aus ne t'afis. » (III, v. 53-54 et 68-70). Alexandre devait se souvenir également du meurtre de Darius, assassiné par deux serfs qu'il avait pris à son service ${ }^{11}$, car, comme l'affirme un soldat du potentat de Perse : «Pire est orguel de serf que venin de crapot» (III, v. 258). On notera que dans ces trois exemples tirés du Roman d'Alexandre la menace que représentent les serfs placés dans l'entourage des puissants est associée à celle du poison. L'ingratitude d'Antipater et Divinuspater est fortement dénoncée dans Le Vengement Alexandre de Gui de Cambrai où il est fait continuellement référence à la condition sociale des deux vassaux félons : «Ce fu mout grans dolors le jor k'il fu traïs / Par les sers de put aire qu'il avoit afrankis. » (v. 279-280). Le poison est l'arme des traîtres qui opèrent secrètement, évitant ainsi l'affrontement avec une victime qu'ils redoutent. Selon la mentalité aristocratique, ces êtres déloyaux ne peuvent être que de basse extraction. Dans le texte d'Alexandre de Paris, Antipater et Divinuspater savent qu'ils n'auront ni la force ni le courage de braver Alexandre. Dans la crainte de représailles, ils choisissent un poison dont l'effet sera retardé ${ }^{12}$. Trahi par ses hommes au sein même de sa cour, dans le cadre familier et festif d'un banquet, Alexandre a donc commis, à l'instar de Darius, une erreur politique fatale.

\section{Le venin de la convoitise}

Tout au long de son parcours à travers l'Asie Mineure, l'Égypte, la Perse ou l'Inde, Alexandre est mû par un désir immodéré de conquêtes. Il ambitionne d'explorer et de conquérir la totalité des terres connues. Il repousse même les limites de celles-ci lorsqu'il franchit les bornes d'Hercule ou se rend aux portes du paradis terrestre $^{13}$. Son ambition, jugée sévèrement par les moralistes, porte une ombre sur sa renommée, faisant de lui l'incarnation de l'hybris. La démesure du conquérant a pu aisément être associée à l'intempérance et a sûrement contribué à l'accuser d'ivrognerie. Cette mauvaise réputation a été véhiculée notamment par Arrien et Plutarque. Au Moyen Âge, on la retrouve sous la plume de Gautier de Châtillon. La soif permanente d'Alexandre, soif qui s'avèrera mortelle lorsqu'il boira le vin empoisonné préparé par Antipater, est aussi la métaphore du désir de conquête et de pouvoir qui l'anime. Dans le Roman d'Alexandre d'Alexandre de Paris, le héros est

${ }^{11}$ Darius III mourut assassiné par ses satrapes. Il fut frappé à mort, sur l'ordre de Bessos, par Satibarzanès et Barsaentès (Arrien, 3, 21, 9-10). Dans les textes issus de la tradition légendaire, les traîtres ne sont plus que deux, Bessos et Ariobarzanès (Epitome, II, 20-21).

${ }_{12}$ «Andui ont porchacié le venim d'un serpent, / Et est de tel nature, se l'estoire ne ment, / Que qant li hom le boit et el cors li descent, / Desq'au nuevisme jor ne bien ne mal ne sent, / Et qant vient au termine, adont li maus li prent / Et au disime jor l'ame du cors li rent. / Itel le quirrent il par apercevement, / Car qant il le beüst, s'il fust mors en present, / Dont fust il conoissant a trestoute la gent / Que il l'eüssent mort par lor enchantement. » (Alexandre de Paris, Le Roman d'Alexandre, III, v. 7824-7833). Le texte se contredit ensuite puisque, lors du banquet fatal, Alexandre ressent les effets du poison dès l'instant qu'il porte la coupe à sa bouche. Pris de malaise, il meurt quelques heures plus tard.

${ }^{13}$ Cette expédition est relatée dans Le Voyage d'Alexandre au paradis terrestre, interpolation au Roman d'Alexandre d'Alexandre de Paris. 
continuellement assoiffé pendant sa traversée des déserts arides. À la recherche d'eau douce, il a les plus grandes difficultés à étancher la soif qui le tourmente; le point d'eau qu'il finit par trouver sera infesté de redoutables hippopotames (III, laisses 59-61), ou cerné de bêtes sauvages (III, laisses 73-86), ou l'eau n'en sera pas potable :

\author{
Dementres que chascuns de herbregier s'argüe, \\ Descent li maines rois de sa mule crenue, \\ Il s'est agenoilliés desor l'erbe menue \\ Por estaindre sa soif qu'il a si grant eüe. \\ Plus iert amere l'eaue, quant li rois l'ot beüe, \\ Que suie ne fauterne n'aluisne ne ceüe. \\ Tel angoisse ot li rois tous li cors li tressue \\ Et voit que sans travail n'en bevroit beste mue, \\ Si l'a a toute l'ost v[e]ee et deffendue. \\ (Alexandre de Paris, Le Roman d'Alexandre, III, v. 1068-1076)
}

L'eau amère dont doit se contenter le Macédonien est comparée à la suie, l'aristoloche, l'absinthe ou la ciguë, substances amères et surtout toxiques. Le poison devient le châtiment métaphorique de la convoitise, vice qui entache la renommée d'Alexandre dans la littérature médiévale. Dans la Prise de Defur, l'interpolateur retravaille ce thème. En effet, dans cet épisode écrit au XIII ${ }^{\mathrm{e}}$ siècle pour être inséré dans la trame de l'œuvre d'Alexandre de Paris, Alexandre est présenté comme un conquérant insatiable; sa démesure est à plusieurs reprises condamnée, notamment par le biais de la parabole de l'œil humain (laisses 57-58) ${ }^{14}$. De surcroît, au cours de son parcours vers Babylone, le Macédonien est confronté de manière réitérée au poison, menace qui vient très clairement sanctionner son avidité. Alexandre lui-même n'établit-il pas la comparaison au début du poème lorsqu'il affirme que «Malvaise covoitise est pire de venin » (v. 551) ? Après avoir quitté la reine Candasse, Alexandre emmène ses hommes sur la route de Babylone. Assoiffés, ils arrivent au bord de la rivière Carengene, mais ne peuvent que constater la toxicité de l'eau : "Tant iert sure et amere et de mauvais pourtrait / Onques beste ne hons n'en pot boire un seul trait.» (La Prise de Defur, v. 1321-1322). Une autre mésaventure particulièrement édifiante arrive peu après à Alexandre. Toujours à la recherche d'eau douce, Alexandre rencontre un vieux paysan et se fait passer auprès de lui pour un simple soldat. L'homme indique à la troupe qui accompagne le Macédonien la direction de la rivière Sapïence mais la met en garde : cette rivière possède des pouvoirs merveilleux; ne peuvent y boire que les hommes vertueux ; elle devient en effet délétère pour les « couvoiteus », les « escars » et les «traïtres

\footnotetext{
${ }^{14}$ Alexandre trouve sur sa route un œil posé sur une pierre. Aristote, qui l'accompagne, lui donne l'explication de cette étrange trouvaille. Cet œil est le symbole de la convoitise du héros qui désire tout ce qu'il voit. Aucun objet n'est assez lourd pour le contrebalancer à moins que l'œil ne soit recouvert d'une étoffe : «[...] oels est de tel diaigne / Quant qu'il voit tout couvoite, fais fu de male ouvraingne. / Tant com cist fu ouvers tout pesa, fer et laingne, / Et quant il fu couvers d'un paile d'entresaingne / Doi besant l'enporterent, çou virent cil castaingne. » (La Prise de Defur, v. 1583-1587).
} 
prouvés » (v. 1362). Tholomer et les deux hommes qui l'accompagnent préfèrent rebrousser chemin quand Alexandre est bien décidé à boire de l'eau. Il reste donc auprès du paysan et fait la preuve, lors de la conversation qui s'engage, de sa grande convoitise. Le vieil homme le rabroue violemment: "Tais toi ! dans couvoiteus, parlés as folement, / $\mathrm{Ne}$ gousteras de l'iaue, retourne isnelement. » (La Prise de Defur, v. 1458-1459). Malgré cette admonestation, Alexandre se dirige vers la rivière, mais l'eau limpide se change immédiatement en liquide putride. Les miasmes qui s'en dégagent lui soulèvent le cœur et obscurcissent sa vue :

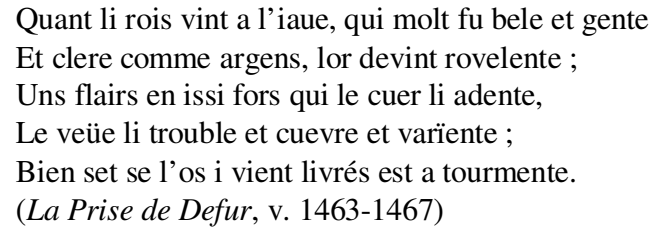

La convoitise d'Alexandre est donc punie dans la Prise de Defur par une menace d'empoisonnement. L'eau devenue «pire de touscin» (v. 1480) constitue le comparant récurrent de son ambition coupable. Dans le Voyage au Paradis terrestre, la même métaphore est employée par Aristote dans une leçon qu'il destine au jeune roi : «Convoitise est el mont molt male enerbeüre, / Avarisce est sa mere, li une et l'autre est sure. » (Le Voyage d'Alexandre au paradis terrestre, v. 251-252).

$\mathrm{Au}$ début du XIV ${ }^{\mathrm{e}}$ siècle, l'évocation de l'empoisonnement d'Alexandre encadre la narration des Vœux du paon qui commence et s'achève sur une déploration de la mort annoncée du Macédonien ; le narrateur impute très clairement cette fin inéluctable à la convoitise du héros et plus précisément à son désir d'ajouter Babylone à la liste de ses conquêtes :

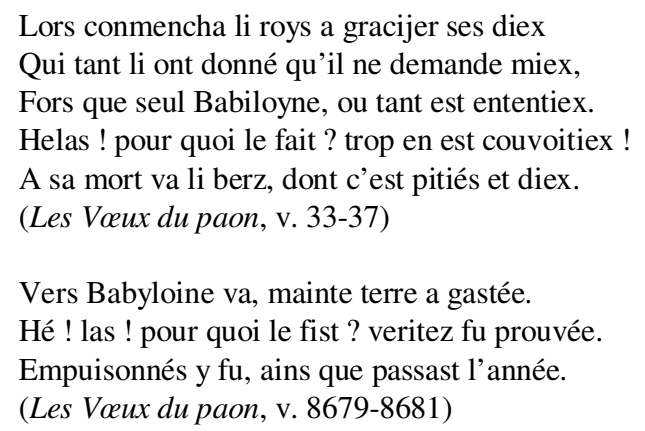

Le terme de «couvoiteus », utilisé par le paysan de la Prise de Defur pour qualifier Alexandre (v. 1458), se retrouve dans le commentaire du narrateur des Voux du paon: «Trop en est couvoitiex!» (v. 36). Le complot d'Antipater et de Divinuspater est ainsi passé sous silence. N'apparaît plus que la condamnation de l'ambition du Macédonien dont l'ultime conquête, Babylone, symbole de superbia, apparaît comme la conquête fatale. L'interpolation des Voux du paon ouvre une parenthèse courtoise dans la destinée du héros, parenthèse qui, par une interruption 
des logiques prévalant dans son parcours, celle de la vengeance et celle de la conquête, permet à Alexandre, mort en sursis, de profiter d'un répit ${ }^{15}$.

\section{La vulnérabilité du roi}

Alexandre, qui est parvenu à repousser les limites de son empire au-delà des bornes du monde connu, qui a soumis les potentats de la Perse et de l'Inde, succombe à une gorgée de vin. Ce paradoxe est souligné par cette réflexion ironique de Gautier de Châtillon : Crescit auara sitis iuueni, sed potio tantam / Comprimet una sitim (Gauthier de Châtillon, Alexandréide, X, v. 200-201) ${ }^{16}$ ( La soif avide du jeune homme croît, mais une seule gorgée étanchera si grande soif. » $)^{17}$. Contenter sa soif, mettre fin à sa quête de pouvoir correspondent pour le héros à une condamnation à mort. Cette caractéristique du destin d'Alexandre est illustrée sur le mode métaphorique dans un épisode du Roman d'Alexandre d'Alexandre de Paris qui relate comment les Grecs parviennent à tuer le Dentirant, ce monstre du désert indien, venu s'abreuver au bord de l'étang où l'armée d'Alexandre a dressé son camp. Cette créature menaçante résiste aussi bien aux coups d'épée, de lance et de hache qu'aux jets de flèches dont elle est criblée. Alexandre conseille alors à ses hommes de la laisser boire. De fait, une fois désaltérée, la bête devient vulnérable et succombe sous les coups (Le Roman d'Alexandre, III, laisses 79-82). Alexandre mourra de la même façon quand, parvenu à Babylone, il sera repu de conquêtes et se sera désaltéré à la coupe des traîtres. Le monstre redoutable, le Dentirant - comment ne pas entendre derrière ce mot celui de tyran ? - est en quelque sorte un double métaphorique et inquiétant du roi omnipotent, qui, comme le dit l'auteur du Voyage d'Alexandre au paradis terrestre par un raccourci saisissant, est « de la mort abevrez » (v. 403).

Le caractère dérisoire de l'instrument responsable de la mort du Macédonien est renforcé par Alexandre de Paris qui, reprenant un élément du récit du PseudoCallisthène, précise que c'est une plume, arme bien inoffensive a priori, qui achève le roi. En effet, après avoir bu le vin empoisonné et ressenti les premiers malaises, Alexandre tente de se faire vomir à l'aide d'une plume :

Puis sailli de la table, la coupe a jus getee,

Por ce que vomir vaut une plume a rovee.

Antipater li fel l'en a une aprestee

Q'il ot molt coiement sous son mantel botee,

Et estoit de venim entoschie et louee.

Il a prise la plume, ne l'a pas esgardee,

Si l'a isnelement en sa bouche boutee ;

Li deerains venins li a la mort donee.

\footnotetext{
${ }^{15}$ Voir à ce sujet H. Bellon-Méguelle, Du Temple de Mars à la Chambre de Vénus. Le beau jeu courtois dans les Vœux du paon, Paris, Champion (Essais sur le Moyen Âge XXXVIII), 2008.

${ }^{16}$ Nous citons le texte latin d'après l'édition: Galteri de Castellione, Alexandreis, ed. M. L. Colker, Padova, Editrice Antenore, 1978.

${ }^{17}$ Nous citons la traduction de l'Alexandréide réalisée par J.-Y. Tilliette. Nous le remercions vivement de nous avoir donné accès à ce travail encore inédit.
} 
Tuit li menbre li falent, la poitrine a enflee.

(Alexandre de Paris, Le Roman d'Alexandre, IV, v. 159-167)

La plume a été au préalable imbibée de poison par Antipater. En l'introduisant dans sa bouche, Alexandre, au lieu de soulager son mal, ne fait que l'aggraver. Alexandre meurt en dilatant sa poitrine. Le terme «enfler», du latin inflare «souffler dans », peut certes décrire le gonflement dû à l'intoxication mais aussi suggérer le dernier souffle du mourant. N'est-il dès lors pas possible de rapprocher cette mort du héros de celle de Roland qui expire dans un ultime et terrible souffle ? Alexandre est un personnage profondément ambivalent. La soif qui le caractérise peut conduire à diverses interprétations. Symbole du désir de conquête du roi, elle permet aussi d'assimiler ce dernier aux héros, tel le martyr de Roncevaux ou celui d'Archamp qui, imitant la passion du Christ, meurt victime de sa soif (Jean, 19, 18) ${ }^{18}$.

\section{Une mort valorisante?}

\section{Le poison et le glaive}

Dans son étude Le crime de poison au Moyen Âge, Franck Collard s'intéresse à l'exemple d'Alexandre le Grand. Il précise que la mort par poison est contraire à l'idéal chevaleresque. Cette mort sournoise, sans effusion de sang, ne correspond pas au topos de la mort héroïque; comment peut-on en effet envisager qu'un chevalier ne meure pas sur le champ de bataille les armes à la main ? D'ailleurs, le héros qualifie lui-même son empoisonnement de «laide mort despite» (Le Roman d'Alexandre, IV, v. 299). Alexandre est ainsi privé de la belle mort assurant aux héros épiques gloire et renommée. Toutefois, le texte d'Alexandre de Paris sousentend que, si les traîtres choisissent d'éliminer le Macédonien en ayant recours au poison, l'arme des lâches, c'est parce que le roi est invincible aux armes. Ainsi Divinuspater conseille son complice: "Rien n'i ferons par force, ja celer nel vos quier; / S'engins ne nos aïde, force n'i a mestier.» (III, v. 7785-7786). La façon dont meurt Alexandre est donc paradoxalement une manière de mettre en valeur la force du héros. Roland aussi, invincible dans la lutte armée, n'est finalement vaincu que par lui-même lorsqu'il se fait éclater les tempes par la violence de son souffle.

L'empoisonnement d'Alexandre ne jette donc pas absolument le discrédit sur le héros dans le roman d'Alexandre de Paris. Il n'en reste pas moins que l'opposition entre le poison, arme vile, et le glaive, arme noble, semble problématique et demeure sous-jacente dans les différents récits relatant la fin du Macédonien. Elle est exposée dans les derniers vers de l'Alexandréide de Gautier de Châtillon, alors que le narrateur s'adresse à Fortune :
[...]
conuerte uenenum
In gladium. satius et honestius occidet armis
Is qui plus deliquit in hiis. sed forsitan armis
Non potuere palam superi quem uincere dirum
Clam potuit uirus. fuit ergo dignius illum

\footnotetext{
${ }^{18}$ Sur ce motif de la soif du héros voir J. H. Grisward, «Les morts de Roland », Mélanges René Louis, Saint-Père-sous-Velay, 1982, p. 417-427.
} 
Occultum sentire nephas quam cedere ferro.

(Galteri de Castellione, Alexandreis, X, v. 210-215)

(Change le poison en épée : il sera plus satisfaisant et plus honorable de tomber sous les armes, pour lui qui a le plus péché par elles. Mais peut-être des dieux furent-ils impuissants à vaincre ouvertement par les armes celui qu'un funeste venin put secrètement vaincre. Il convenait donc qu'il eût à subir un crime sournois plutôt qu'à tomber sous le fer) ${ }^{19}$

Certains auteurs médiévaux sont parvenus à neutraliser cet antagonisme entre poison et glaive grâce au pouvoir de la métaphore. Ainsi chez Thomas de Kent ${ }^{20}$, auteur anglo-normand qui écrit dans les mêmes années qu'Alexandre de Paris ${ }^{21}$, le poison pourfend les entrailles d'Alexandre à la manière d'une arme blanche :

Li beiveres fu ague, e si chet trenchantment;

Tut li ret le ventrail, a poi k'il ne fent.

(Thomas de Kent, Le Roman d'Alexandre, C 182-183)

Chez Jean Wauquelin ${ }^{22}$, trois siècles plus tard, les atteintes du poison sont comparées de la même manière à celles de l'épée :

[...] il mist la couppe a sa bouche et but. Mais veritablement, tantost qu'il ot but, il s'enclina a la dextre partie de son siege, car il lui sembla parfaictement que on lui eust le cuer perchié de une espee.

(Jehan Wauquelin, Les Faicts et les conquestes d'Alexandre le Grand, 254, 53-57)

Dans ce roman du $\mathrm{XV}^{\mathrm{e}}$ siècle, Alexandre reçoit le présage de sa mort par un songe dans lequel Cassandre, le fils d'Antipater, le transperce d'une épée. Ces mises en équivalence du poison et de l'épée laissent deviner une volonté chez certains auteurs de la tradition alexandrine d'ennoblir la mort du héros qu'ils entendent célébrer.

\section{Henri VII, un nouvel Alexandre}

Il est un exemple appartenant à l'histoire du début du XIV siècle dans lequel l'empoisonnement d'Alexandre est utilisé à des fins d'éloge. Au fil des récits historiographiques et littéraires qui lui furent consacrés, l'empereur germanique Henri VII, prétendument empoisonnée ${ }^{23}$ en août 1313, fut élevé au rang de nouvel

\footnotetext{
${ }^{19}$ Traduction de J.-Y. Tilliette, à paraître.

${ }^{20}$ Thomas de Kent, Le Roman d'Alexandre ou Le Roman de toute chevalerie, trad., prés. et notes de C. Gaullier-Bougassas et L. Harf-Lancner avec le texte édité par B. Foster et I. Short, Paris, Champion (Champion Classiques, série Moyen Âge), 2003.

${ }^{21}$ Thomas de Kent écrivit le Roman de toute chevalerie entre 1175 et 1185 et Alexandre de Paris rédigea son roman entre 1180 et 1185 .

${ }^{22}$ Jehan Wauquelin, Les Faicts et les Conquestes d'Alexandre le Grand, éd. crit. par S. Hériché, Genève, Droz (TLF 527), 2000.

${ }^{23}$ Les historiens s'accordent aujourd'hui à dire que l'Empereur serait mort en réalité de la malaria (comme Alexandre !). Sur la rumeur d'empoisonnement qui entoura la disparition du
} 
Alexandre. La similitude supposée entre les circonstances de leur mort fut à l'origine de cette glorieuse comparaison. Dans le contexte des luttes opposant Guelfes et Gibelins, la rumeur de l'empoisonnement d'Henri VII, venu en Italie pour être couronné, se répandit rapidement et fut utilisée comme instrument de propagande politique par les Gibelins, partisans de l'Empereur. Selon eux, le crime aurait été commandité par Robert d'Anjou, roi de Naples, qui soutenait le parti guelfe, et exécuté par un frère jacobin pendant la messe grâce à une hostie empoisonnée ou du vin d'ablution. Henri, dans la fleur de l'âge ${ }^{24}$, aurait donc été traîtreusement assassiné alors qu'il avait atteint aux plus hautes dignités. Dans ces circonstances le rapprochement entre les deux monarques était aisé à établir. Cela fut fait notamment par le copiste du manuscrit W des Voux du paon (Paris, BNF, fr. 12565). Dans un épilogue devenu célèbre, ce dernier évoque la mort de l'Empereur ainsi que celle de son grand-oncle, Thibaut de Bar, qui apparaît dans ces lignes comme le commanditaire du poème :

Tybaus fu mors a Ronme avoec .i. Lembourgis

Qui empereres ert, si ot a nom Henris,

De Luxembourc fu quens et chevaliers eslis.

Jacobin preëcheur, (qui soient tous honnis !)

Le firent par poison morir, dont il est pis

A tous bons crestiens, et a tout par paÿs.

(Les Veux du paon, v. 8773-8778)

Cette évocation de la mort d'Henri VII survient juste après les regrets que le narrateur exprime au sujet de la disparition prématurée d'Alexandre. Nous pensons qu'en écrivant son épilogue, le copiste de W avait en mémoire le texte des Voeux de l'épervier de Simon de Marville $(1313)^{25}$. De manière significative, il choisit d'évoquer la mort de l'Empereur dans une laisse monorime en i sur le modèle de celle qui relate le même crime dans les Voux de l'épervier (laisse XXVI) ${ }^{26}$. Cette dernière œuvre est elle-même une réécriture des Voux du paon; la filiation est très clairement signalée par Simon de Marville dès les premiers vers de son poème,

monarque, nous renvoyons aux travaux récents de Franck Collard: outre les pages qu'il y consacre dans Le crime de poison au Moyen Âge, op. cit., ses deux articles : «L'empereur et le poison : de la rumeur au mythe. Les enjeux historiographiques, politiques et idéologiques du prétendu empoisonnement d'Henri VII en $1313 »$, Médiévales 41, 2001, p. 113-131 et «Jacobita secundus Judas. L'honneur perdu des Prêcheurs après la mort d'Henri VII », Religions et mentalités au Moyen Âge. Mélanges Hervé Martin, Rennes, PUR, 2003, p. 221 235.

${ }^{24}$ Henri est mort à trente-cinq ans, âge qui rapproche également sa destinée de celle d'Alexandre.

${ }^{25}$ Nous renvoyons à ce propos à l'étude intitulée «La datation et l'attribution des Vœux du paon : des évidences à revoir?», placée à la fin de notre ouvrage Du Temple de Mars à la Chambre de Vénus, op.cit, p. 471-488.

${ }^{26}$ Les Voux de l'épervier, hrsg. von G. W. und F. Bonnardot, dans Annuaire de la Société d'histoire et d'archéologie lorraine, sixième année, 1894, p. 177-280. 
calqués sur les vers liminaires de son hypertexte ${ }^{27}$. Un rapport complexe d'intertextualité existe donc entre les deux textes: les Vøux du paon ont servi de modèle aux Voux de l'épervier, l'œuvre historiographique a en retour inspiré la rédaction d'un épilogue contenu dans un manuscrit tardif de l'œuvre alexandrine. L'épopée d'actualité de Simon de Marville a pour sujets principaux l'accession au pouvoir d'Henri et son empoisonnement par deux moines jacobins. L'empereur y est présenté comme un grand conquérant, capable de soumettre en moins d'un an deux royaumes (v. 31-33), un roi bien-aimé, assuré de la loyauté de ses hommes. Dans la première partie du texte, Thibaut de Bar remémore lors d'un banquet la cérémonie des vœux sur le paon, épisode central et éponyme de l'interpolation alexandrine. L'évêque suggère de s'en inspirer en choisissant comme oiseau l'épervier de Wallerand, le frère d'Henri. Les douze pairs d'Allemagne, tels les douze pairs de Macédoine, participent à la cérémonie et prononcent des voux de bravoure à l'instar des chevaliers d'Éphèse. Peu de temps après, Henri se rend en Italie et c'est lors d'un séjour à Buon Covento près de Sienne qu'il est empoisonné. Le texte insiste sur les liens d'affection qui unissaient Henri à ses meurtriers. Ce n'est certainement pas un hasard si les jacobins, coupables du forfait, sont deux dans le texte de Simon : tels Antipater et Divinuspater, ils jouissent de la confiance et de l'attachement du souverain (Les Voux de l'épervier, v. 508-511). Les symptômes de l'intoxication sont les mêmes que ceux présentés par Alexandre; l'empereur d'Allemagne voit sa beauté se flétrir et sa peau noircir sous l'effet du venin : «cil que fut blan com flour, est tout tantost nercis » (Les Voux de l'épervier, v. 504) à l'instar d'Alexandre dont la bouche «plus blanche que n'est oef de geline» devient «plus noire qu'escorce d'aubespine » (Alexandre de Paris, Le Roman d'Alexandre, IV, v. 660-661). Comme Alexandre encore, Henri, ressentant les premiers malaises, se retire dans sa chambre où il est rejoint par ses compagnons. L'auteur des Veux de l'épervier insiste sur la grandeur d'âme de l'Empereur qui refuse de vomir pour ne pas rendre l'hostie sacrée et conserver le corps de Jésus-Christ en lui et qui pardonne à ses meurtriers, leur permettant d'éviter par la fuite le châtiment qui les attend. Henri, loué pour sa grande largesse (v. 485), est comparé explicitement dans les derniers vers du poème à Alexandre le Grand :

Ha ! Jhesu Crist sire, per queille desamour

Avéz heus mis a mort le muedre empereour

Que fut pues Alixandre le lairge donneour?

(Les Voux de l'épervier, v. 558-560)

\footnotetext{
${ }^{27}$ « Aprés ce qu'Alixandres ot Dedephur conquis

Et a force d'espée occis le duc Melchis,

Floridas en mena, si marïa Dauris ;

Chevaucha li bons roys, liés et gais et jolis. » (Les Voux du paon, v. 1-4)

« Aprés ce que Hanrey olt deden Mets conquis,

Et a force de bras de lour guerre acomplis,

Et enver les bourgois accourdez et paix mis,

S'en departit li cuen, biaul, liéz et jollys. » (Les Voux de l'épervier, v. 1-4)
} 
Le temps que met le poison à agir lui permet de distribuer ses terres et de régler sa succession à la manière de son illustre prédécesseur (Les Voux de l'épervier, v. 540$542)^{28}$. Henri VII, souverain exemplaire, trépasse donc comme Alexandre. Aux yeux des auteurs médiévaux, c'est tout à son honneur.

\section{La sagesse du roi}

Dans le Roman d'Alexandre d'Alexandre de Paris, le roi n'a finalement d'autre choix que de se soumettre au destin fixé par les dieux. Pourtant, une tension existe chez le personnage entre acceptation et accablement. Lorsque les voix émanant des arbres du Soleil et de la Lune prédisent à Alexandre sa mort prochaine, celui-ci est profondément abattu. Raisonné par ses hommes, il parviendra à réconforter lui-même ses compagnons les plus affligés :

Por sa gent esbaudir se prent a conforter ;

Puis a demandé l'eaue s'est assis au disner.

Et a dit a ses homes : « Cest duel laissiés ester,

La mors n'est pas teus chose que on puist eschiver,

Ains la convient a tous soufrir et endurer,

Car deci a mon terme ne m'estuet riens doter. »

(Alexandre de Paris, Le Roman d'Alexandre, III, v. 3862-3867)

À ce moment du récit, ces paroles de sagesse sont celles d'un bon chef qui se domine et parvient à cacher sa peur pour rasséréner ses hommes. Cependant, à partir du moment où il reçoit l'oracle funeste, Alexandre ne parvient pas à se départir de son angoisse. Lors du banquet à Babylone, il tente en vain de se préserver de l'empoisonnement prédit, nous l'avons vu, en ordonnant à ses serviteurs de couper leurs manches pour qu'ils n'y dissimulent pas de poison. Sur son lit de mort, Alexandre, bien que réduit au désespoir, saura pourtant trouver les mots pour consoler ses hommes. Une fois de plus, il fait preuve d'un attachement et d'une sollicitude exemplaires envers eux. S'il regrette de mourir, c'est avant tout parce qu'il ne veut pas les quitter et qu'il lui est insupportable de les voir souffrir. Le Roman d'Alexandre poursuit un projet didactique qui l'apparente fortement à un miroir du prince. À cette fin, il met en avant les qualités politiques du roi de Macédoine : Alexandre, même dans l'agonie, se comporte comme un bon chef.

Thomas de Kent poursuit un projet sensiblement différent. Il fait d'Alexandre un roi monothéiste converti au judaïsme ${ }^{29}$, dont la mort sert d'illustration à la sagesse

\footnotetext{
${ }^{28}$ Renate Blumenfeld-Kosinski a bien mis en évidence les parallèles établis par Simon de Marville entre Henri de Luxembourg et Alexandre le Grand dans les Voux de l'épervier dans son article: "Historiography and Matière Antique. The Emperor Henry VII as a New Alexander in the Fourteenth-Century Voeux de l'Epervier", Medievalia et Humanistica, New Series 14, 1986, p. 17-35.

${ }^{29}$ Thomas de Kent raconte comment, après s'être rendu à Jérusalem, Alexandre adopte la religion juive (Le Roman de toute chevalerie, laisses 187-193).
} 
du héros, capable d'accueillir avec équanimité la mort voulue par Dieu ${ }^{30}$. Alexandre montre une sérénité remarquable et, ressentant les premiers effets du poison, trouve la force de prononcer un discours édifiant sur la vanité des biens de Fortune et sur la précarité de la vie humaine (Thomas de Kent, Le Roman de toute chevalerie, laisses 537-538):

«Seignurs, fet Alisandre, lessez le suspir.

Ne profist a nul home qe ses chevelz detir

Ou ke se[s] palmes bate ou se[s] draps decir,

Car ceo est necessaire et ne poet faillir

Qe quant qe vit el siecle en covient morir.

Ore $\mathrm{i}$ ad meint home qui nule rien desir

Fors qe longe vie en eise [e] a pleisir

E bones destinez, pleisantes a loisir,

E quant plus s'en travaille e mieutz quide joir,

Amere mort le prent e le fet departir,

Car fortune le desceait par son resortir. »

(Thomas de Kent, Le Roman de toute chevalerie, v. 7917-7927)

Philosophe, Alexandre prend de la hauteur et porte un regard critique sur les hommes qui, à son exemple, ont passé leur vie à convoiter le pouvoir et les biens terrestres auxquels ils doivent renoncer au moment de mourir. Tel son maître Socrate, Alexandre s'administre lui-même le poison; comme lui, il accepte impassiblement son sort.

\section{L'homme va mourir}

\section{Une mort annoncée}

Dans la vita d'Alexandre le Grand, plusieurs signes manifestes annoncent sa mort prématurée; parmi eux, l'oracle des Arbres du Soleil et de la Lune et la naissance d'un enfant monstrueux à Babylone peu de temps avant la cérémonie du couronnement d'Alexandre. D'autres événements jalonnant le parcours du conquérant peuvent être lus comme les tristes présages de son empoisonnement. Ainsi, dans le Roman d'Alexandre d'Alexandre de Paris, le jour fatal est annoncé de manière tragique par certaines mésaventures au cours desquelles Alexandre est confronté de manière anticipée aux affres de la mort par poison. La branche I relate l'épisode bien connu du bain d'Alexandre dans une eau glacée. Après avoir plongé dans la rivière Nidèle ${ }^{31}$, Alexandre est pris de malaise. Atteint de mutisme, il devient

${ }^{30}$ Cf. Introduction au Roman d'Alexandre de Thomas de Kent par C. Gaullier-Bougassas et L. Harf-Lancner, op. cit., introduction, p. LXII-LXV: «Le sage: l'acceptation de la loi divine».

${ }^{31}$ Chez Quinte-Curce, conformément aux autres récits historiques, Alexandre tombe malade en se baignant dans le Cydnos, fleuve de Cilicie (Histoires, III, 4, 5-6). Dans le roman du Pseudo-Callisthène, Alexandre souffre de maux de tête après s'être baigné dans le fleuve Océan (Pseudo-Callisthène, Le Roman d'Alexandre, trad. et éd. par A. Tallet-Bonvalot, Paris, Flammarion, 1994, p. 98-99). 
livide, éprouvant des souffrances similaires à celles causées plus tard par son empoisonnement. Il demande à ses hommes de l'emmener dans sa tente où ces derniers, convaincus que le roi va mourir, manifestent leur deuil de la manière la plus pathétique (I, laisses 116). Cet épisode de la maladie d'Alexandre est, comme le veut la tradition, immédiatement suivi du complot de Darius. Le roi de Perse tente de corrompre le médecin d'Alexandre, afin qu'il empoisonne le roi. Alors que dans les textes antiques le médecin loyal refuse, chez Alexandre de Paris il commence par accepter avant de se raviser in extremis et de soigner Alexandre (I, laisses 117-120). Le roi échappe donc de peu à la mort dans le roman médiéval, où l'épisode ne représente plus une illustration de la loyauté des hommes du Macédonien et de la confiance que ce dernier leur fait, mais une mise en garde contre la menace que peuvent constituer les familiers du roi.

Certes Alexandre meurt trahi par ses serviteurs, mais cette mort répond avant tout à une décision divine. Dans le dernier chant de l'Alexandreide, Gautier de Châtillon met en scène les démarches entreprises par les puissances surhumaines pour punir Alexandre et l'arrêter dans sa course. Son ambition exaspère Nature qui descend aux Enfers et incite le Leviathan à mettre en œuvre la trahison et le meurtre par poison du héros. Dans le Roman d'Alexandre, Alexandre excite pareillement la colère des dieux qui condamnent l'hybris du conquérant. Plusieurs incidents signalent la désapprobation des forces supérieures. Après avoir dépassé les bornes d'Arthur, avatars des bornes d'Hercule, s'aventurant ainsi plus loin qu'aucun être humain ne l'avait fait auparavant, surpassant même les dieux par son audace, Alexandre et ses hommes se trouvent enfermés dans le Val périlleux où ils subissent de terribles épreuves. Alors que l'armée ne trouve pas d'issue et tourne en rond dans la vallée funeste, Alexandre y voit le signe d'une malédiction : "Li dieu nos veulent mal, tuit somes deceü, / De ci n'istront ja mais ne joene ne chanu. » (III, v. 25142515). Une inscription gravée dans du marbre indique à Alexandre que leur libération ne se fera qu'au prix de la vie de l'un d'eux. Alexandre est alors prêt à se sacrifier. Resté seul dans le lieu désolé, le roi doit affronter le tumulte des éléments, respirer la puanteur qui emplit le val, voir la terre s'enflammer et trembler, attendre enfin la mort, agité par l'angoisse et assailli par des créatures infernales. Il survit grâce à l'aide d'un démon qu'il libère de la pierre sous laquelle il était prisonnier. Les forces infernales accordent un sursis au conquérant qui sera pourtant bientôt rattrapé par son destin. Pendant sa traversée de l'Inde, Alexandre reçoit d'autres avertissements. Son armée subit de lourdes pertes à cause des cataclysmes qui s'abattent sur elle : feu tombé du ciel, neige, pluies diluviennes. Les hommes en sont sûrs, ces catastrophes sont causées par les divinités qu'ils ont outragées : «Libers est irascus, / Ou il ou Erculés font ore ces vertus, / Par la consence as dieus nos est cis maus venus. » (III, v. 3158-3160). Les deux divinités, dont les bornes n'ont pas été respectées par l'armée qui s'est aventurée au-delà, sont à l'origine d'un autre maléfice. Elles ont empoisonné une grotte dans laquelle Alexandre fait entrer quatre de ses hommes qui, peu après, sont retrouvés morts. Le roi demande une explication de ce mystère à deux vieux Indiens :

L'un des deus avant l'autre a forment conjuré,

Que de cele mervelle qui l'a si effreé

Li dïent ambedui toute la verité : 
S'en la cave a fantosme, serpent ne enferté

Qui si soutainement a cest lieu deserté.

Li dui viellart respondent, qui sont de grant aé,

Que Artus et Libers par lor grant deité

Entoschierent le lieu si l'ont enfantosmé.

(Alexandre de Paris, Le Roman d'Alexandre, III, v. 3239-3246)

Les dieux irrités savent, quand il le faut, user du poison. Voilà Alexandre prévenu ! $\mathrm{Au}$ sortir de la forêt des filles-fleurs, Alexandre et ses compagnons traversent un vallon où ils essuient une pluie de sang. Alexandre interprète une nouvelle fois ce prodige apocalyptique comme un signe de la colère divine: «Segnor, fait Alixandres, par le mien ensïent / Cis signes senefie molt grant destruiement. / Li dieu nos volent mal, trop alons folement. » (III, v. 3582-3584). Alexandre comprend qu'il est condamné par un arrêt divin; de nombreux signes avant-coureurs lui font prendre conscience de la fatalité implacable qui pèse sur lui. La connaissance de l'imminence de sa mort donne une dimension tragique à cet homme qui poursuit son chemin et qui, sans renoncer à ses ambitions, tente d'accomplir ses projets tout en se sachant condamné.

\section{L'ironie du sort}

Dans le Roman de toute chevalerie de Thomas de Kent, Alexandre parvient à soigner ses hommes empoisonnés par les flèches des Indiens après avoir appris grâce à un songe quelle herbe serait un contre-poison ${ }^{32}$ (laisses : 346-347). Le héros ne pourra pourtant neutraliser le venin qui lui sera administré par la suite. Amère ironie. De même, dans le Roman d'Alexandre d'Alexandre de Paris, le sort veut qu'au cours de ses explorations, à plusieurs reprises, Alexandre ait à portée de la main l'antidote qui pourrait le sauver de son empoisonnement, sans savoir s'en emparer ${ }^{33}$. Ce sont d'abord les os du Dentirant, monstre qui, une fois abattu par les hommes d'Alexandre, est dépecé. On récupère sa chair et sa peau qui servira de tapis pour les divertissements des chevaliers. Ses os seuls sont jetés à l'eau alors que, réduits en poudre, ils constitueraient un puissant remède, guérissant les blessures et rendant invulnérable pendant une année celui qui en absorberait :

Plus en vaut l'ossemente de quatorze cités ;

Dieus ne fist chevalier, tant soit el cors navrés,

S'il en avoit beü ne fust sempres sanés

$\mathrm{Ne}$ ja puis en tout l'an n'avroit mal en ses les.

De ce fu Alixandres molt malement menés

Que les os de la beste ont en l'eaue getés.

(Alexandre de Paris, Le Roman d'Alexandre, III, v. 1406-1411)

\footnotetext{
${ }^{32}$ On trouve la source de cet épisode chez Justin, Abrégé des Histoires Philippiques de Trogue Pompée, livre XII, 10 et Orose, Histoires contre les païens, livre III, 19, 11.

${ }^{33}$ L'incapacité d'Alexandre à bénéficier des remèdes qu'il trouve sur sa route est un motif récurrent du roman d'Alexandre de Paris. On pourrait citer notamment le cas des pierres guérisseuses, prescrites pour les blessures à l'arme blanche, qu'Alexandre trouve dans le désert indien et regrette de ne pouvoir emporter avec lui (III, laisses 85).
} 
Alexandre apprend ensuite l'existence de trois fontaines merveilleuses ${ }^{34}$, la Fontaine de jouvence, la Fontaine d'immortalité qui n'apparaît qu'une fois par an, et la Fontaine de résurrection. Cette dernière est découverte par le cuisinier d'Alexandre qui voit des poissons qu'il avait fait cuire reprendre vie en tombant dans l'eau de celle-ci. Alexandre décide de préserver cette merveille en scellant la fontaine et en y construisant une tour. Détenteur de ce secret, il ne pourra pourtant en bénéficier luimême (III, laisses 173-174). La Fontaine d'immortalité lui échappe également puisque c'est Énoch, un riche seigneur au nom prédestiné, qui la trouve avant lui et s'y baigne, empêchant ainsi le roi de pouvoir y accéder avant une année. Furieux, Alexandre le fait emmurer vivant (III, laisses 176-178). Enfin, le roi trouve la Fontaine de jouvence dans laquelle plusieurs de ses vieux compagnons se baignent et retrouvent leur jeunesse. Alexandre est ravi de ce spectacle, mais repart sans emporter de cette eau merveilleuse dont le narrateur nous explique les pouvoirs antipoison :

Onques Dieus ne fist home, se la avoit esté,

De venin ne de poudre tant fort envenimé,

S'un petit i avoit dormi et reposé

Et eüst de cele eaue un petitet gousté,

Ne s'en alast halegres et trestous en santé.

(Alexandre de Paris, Le Roman d'Alexandre, III, v. 3647-3651)

Lorsqu'il fait ces découvertes qui pourraient lui sauver la vie, Alexandre n'a pas la prescience du danger qu'il court. Il l'apprendra ensuite grâce aux oracles des Arbres du Soleil et de la Lune qui lui révéleront l'empoisonnement qui l'attend. Malgré ses prétentions et son désir d'atteindre à la divinité, Alexandre n'en demeure pas moins un homme avec ses déficiences et ses faiblesses.

\section{L'humanité d'Alexandre}

Alexandre, guerrier invincible et roi exemplaire, n'échappe pas à l'angoisse existentielle que peut ressentir tout homme qui prend conscience de la précarité de son existence. Lucide sur la brièveté du temps qui lui reste à vivre, Alexandre a terriblement peur de la mort. Alexandre de Paris décrit avec un grand sens du pathétique les symptômes de l'angoisse ressentie par le Macédonien. Dans le Val périlleux, terrifié, il pâlit et change de visage :

La paor quil destraint li change le viaire,

Car qui de mort se crient bien est drois qu'il i paire,

Tous en est enpalis, car il ne set que faire.

(Alexandre de Paris, Le Roman d'Alexandre, III, v. 2716-2718)

\footnotetext{
${ }^{34}$ Sur ce motif, largement développé par Alexandre de Paris, voir L. Harf-Lancner, «La quête de l'immortalité : Les fontaines merveilleuses du Roman d'Alexandre d'Alexandre de Paris », dans Sources et fontaines du Moyen Âge à l'Âge baroque, Actes du colloque tenu à l'Université Paul-Valéry (Montpellier III) les 28, 29 et 30 nov. 1996, Paris, Champion (Colloques, congrès et conférences sur la Renaissance XII), 1998, p. 31-45.
} 
Après avoir reçu la prédiction de son empoisonnement par les Arbres du Soleil et de la Lune, son teint noircit, une grande faiblesse l'envahit au point de ne plus tenir sur ses jambes et de ne plus pouvoir parler:

Li rois ot la parole si tint le chief en bas,

Fremist et devint noirs et de parler fu quas,

Ne pot sor piés ester, tant fu de paor las.

(Alexandre de Paris, Le Roman d'Alexandre, III, v. 3808-3810)

Enfin, lorsqu'il apprend sa mort prochaine par un vieux sage qui interprète le présage de l'enfant monstrueux né à Babylone, le souffle lui manque, il transpire abondamment, tour à tour fiévreux et transi de froid :

Se li rois a paor n'est mie de mervelle ;

Devant lui voit celui qui sa mort li conseille

$\mathrm{Ne}$ de riens ne li dit qui la li apareille.

Il a si grant angoisse que pas des ieus ne ceille,

$\mathrm{Ne}$ nus aspiremens de lui ne descoreille.

Il se vait acouter sous l'ombre d'une treille,

Ne fust pas si molliés de l'eaue d'une seille

Com il est de suor sous la porpre vermeille.

Une eure a chaut li rois si li rougist la face,

Autre eure devint noirs si froidist comme glace.

(Alexandre de Paris, Le Roman d'Alexandre, IV, v. 33-42)

Ces signes de la détresse d'Alexandre sont comparables à ceux qui accompagnent son agonie. Le noircissement de la peau ${ }^{35}$ et le mutisme notamment, qui caractérisent l'angoisse d'Alexandre, sont précisément décrits lors de son empoisonnement. Tout se passe comme si le héros devait vivre sa mort plusieurs fois. Vulnérable, il doit faire à plusieurs reprises la douloureuse expérience des limites imposées par son corps.

On peut voir une volonté de dépasser ces limites corporelles dans la tentative de suicide d'Alexandre. Sentant les effets mortels du poison, le roi décide de se jeter dans l'Euphrate, mais en est empêché par l'intervention de sa femme Roxane. Cet épisode est relaté dans la plupart des biographies légendaires du héros ${ }^{36}$. L'explication la plus souvent donnée à ce geste désespéré est le désir qu'aurait ressenti Alexandre de se soustraire à la vue de ses hommes, soit pour leur épargner

\footnotetext{
${ }^{35}$ Franck Collard explique que le changement de couleur de la peau, et à plus forte raison son noircissement, est tenu au Moyen Âge comme le signe indubitable de l'absorption d'un toxique. Cf. Le crime de poison, p. 79.

${ }^{36}$ Cet épisode est relaté notamment dans le Roman d'Alexandre du Pseudo-Callisthène (p. 153-154); Arrien le mentionne pour le rejeter comme une invention (Anabase, VII, 27, 3). Au Moyen Âge, on le retrouve chez Alexandre de Paris, mais aussi chez Jehan Wauquelin (Les Faicts et les Conquestes d'Alexandre le Grand, 256).
} 
du chagrin $^{37}$, soit pour préserver sa gloire ${ }^{38}$. Alexandre de Paris ne donne pas de raison claire à la décision d'Alexandre. Juste avant de relater son geste, il indique seulement qu'Alexandre ne peut supporter de voir ses hommes se lamenter, ce qui laisse penser que l'auteur français adopte la première explication. En décidant de se jeter dans l'Euphrate, Alexandre tente de remplacer en quelque sorte l'absorption du venin par sa propre incorporation dans l'eau du fleuve, fleuve du jardin d'Éden d'où provient l'eau merveilleuse de la Fontaine de jouvence aux vertus thérapeutiques (Alexandre de Paris, Le Roman d'Alexandre, III, v. 3680-3681). Le poison lui fait ressentir de manière tragique les limites imposées par son enveloppe charnelle qui gonfle et se fend : «Li bel oel de son chief commencent a torbler, / Li nes a acorcier et la bouche a crever » (IV, v. 1409-1410). En se jetant dans l'Euphrate, il pouvait espérer sortir de ce douloureux carcan, le dépasser pour se fondre dans l'immensité du fleuve.

\section{Conclusion}

L'empoisonnement d'Alexandre est une mort annoncée qui donne à la destinée du héros une forte dimension tragique. Cette menace hante la littérature française consacrée à Alexandre, qui présente le héros comme profondément ambivalent. Le motif du poison dans les poèmes qui lui sont consacrés l'est également: la menace constante que constitue pour le personnage la rencontre de liquides délétères est une façon de dénoncer sa trop grande convoitise. Une forte réprobation pèse sur ce personnage marqué par la démesure et vaincu par des serfs. L'empoisonnement du conquérant se trouve entaché d'une condamnation morale. Un soupçon, s'insinuant dans les textes, pèse sur la mort d'Alexandre : celui d'une punition divine. Paradoxalement, l'empoisonnement du héros a pu être perçu comme un élément valorisant de sa destinée, faisant de lui un martyr, victime de l'ignominie des traîtres; tel est le cas en particulier dans les textes qui identifient Henri VII au Macédonien. Dans sa quête de domination absolue, Alexandre est entravé par les limites que lui impose son corps de mortel. Guerrier invincible, héros de l'illimité, Alexandre est aussi un personnage profondément humain qui trouve dans la mort par poison le terme de son ambition, un héros métaphysique qui connaît l'heure de sa

${ }^{37}$ C'est l'explication que donne Alexandre à Roxane dans la réécriture de Jehan Wauquelin : «Et pource vouloye je parvenir a ma fin si priveement afin que, quant il ne me trouvassent, il en fussent moins dolans et moins courrouciez. » (256, 11. 29-32).

${ }^{38}$ Cf. Arrien : «Alexandre était sorti pour aller se jeter dans l'Euphrate, afin de disparaître de la vue des hommes, et ainsi d'accréditer auprès de la postérité la croyance qu'il était né d'un dieu et qu'il était retourné auprès des dieux; mais que, retenu par elle, il avait dit en gémissant qu'elle le privait de la gloire immortelle d'être né dieu. » (Anabase, VII, 28, 3). 
mort et qui en est maintes fois averti. Privé de la belle mort assurant aux nobles guerriers une gloire éternelle, Alexandre, victime de «laide mort», devient un exemple à méditer sur la nécessaire humilité qu'impose aux hommes leur condition.

Hélène Bellon-Méguelle Université de Genève 